\title{
Effects of Ultrasonic Welding on Nanocrystalline Ag-W Investigated with 30kV Transmission Kikuchi Diffraction (tKD) and 300kV STEM SE Imaging
}

\author{
Donovan N. Leonard ${ }^{1}$, Austin A. Ward ${ }^{2}$, Matthew R. French ${ }^{2}$, Zachary C. Cordero ${ }^{2}$ and Samuel R. \\ Cross $^{3}$. \\ 1. Oak Ridge National Laboratory, Oak Ridge, TN, USA \\ 2. Materials Science and NanoEngineering, Rice University, Houston, TX, USA \\ 3. Xtalic Corporation, Marlborough, MA, USA
}

Nanocrystalline alloys have high hardness values [1], excellent wear resistance [2], and other unique mechanical properties that make them ideal for structural applications [3]. However, because these materials have highly non-equilibrium microstructures that tend to coarsen at elevated temperatures [4], and because most welding processes involve a large thermal excursion, it is challenging to retain the structure of nanocrystalline materials when they are being joined. This inability to weld nanocrystalline metals without affecting their structure is a major obstacle that has prevented their widespread use.

In order to overcome the challenges with welding nanocrystalline materials, we have explored joining nanostructured foils using a low-temperature, solid-phase welding process termed ultrasonic welding. Unlike traditional fusion-based welding processes which join workpieces by melting them, ultrasonic welding bonds workpieces using only plastic deformation. During an ultrasonic weld, there is some frictional heating at the weld interface. However a typical weld lasts less than $1 \mathrm{~s}$ and the maximum temperature in the weld zone is normally less than $50 \%$ of the melting temperature of feedstock. Thus, it is expected that ultrasonic welding can preserve the grain structure of nanocrystalline feedstock.

In this study we used ultrasonic welding to join $\mathrm{Cu}$ foils uniformly coated with a nanocrystalline $\mathrm{Ag}$ alloy (Ag-5W, at\%). Next, we used a combination of 30kV transmission Kikuchi Diffraction (tKD), $300 \mathrm{kV}$ scanning transmission electron microscopy (STEM) secondary electron (SE), bright field (BF), annular dark field (ADF) imaging modes and X-ray mapping to compare the structure of the as-received material with that of the welded material. By correlating these electron microscopy techniques, we clarified the effects of ultrasonic welding on the grain size distribution, the crystallographic texture, and the solute distribution.

Figure 1A is a STEM SE micrograph of the as-received Ag foil. Stereological measurements on the asreceived foils gave a mean circular equivalent grain size of $18 \mathrm{~nm}$. Further, EDS measurements showed that the $\mathrm{W}$ and $\mathrm{O}$ were distributed uniformly throughout the Ag. The STEM SE and ADF micrographs, Fig. 1B and Fig. 1C respectively, of the welded material shown revealed dramatic effects of the welding process on foil nanostructure. In contrast with the equiaxed grains seen in the as-received material, grains in the welded material are clearly elongated, with their major axes roughly parallel to the sonotrode oscillation direction. Also evident in Figure 1B are spherical particles with a mean diameter of $90 \mathrm{~nm}$. X-ray maps of these particles, shown in Figure 2, determined the presence of $\mathrm{W}$ and $\mathrm{O}$, and once quantified, indicated they were $\mathrm{WO}_{2}$ dispersoids. Since these particles were not present in the asreceived material, they must have precipitated during the welding process. The same FIB lamella was used for tKD and STEM/EDS and is pictured in Figure 3A. The inverse pole figure (IPF) of Figure 3B shows relative grain orientations after welding and also clearly shows the grain elongation. Further, the welded material has a mean grain size of $625 \mathrm{~nm}$, meaning the grains grew by an order of magnitude 
during the weld. Inspection of the grains revealed that they were mainly free of dislocations, suggesting there was dynamic recovery and recrystallization.

It is noted that both the $\mathrm{WO}_{2}$ particle size and the grain size vary with distance from the weld interface, and that the particle size and the grain size are roughly proportional, in agreement with the Zener relation. The effects of the ultrasonic welding on the nanostructured feedstock are attributed to frictional heating inside the weld nugget, and can be related to the process parameters through classical models of coarsening and grain growth [5].

References:

[1] M.A. Meyers, A. Mishra, D.J. Benson, Progress in materials science, 51 (2006) p. 427.

[2] T.J. Rupert, W. Cai, C.A. Schuh, Wear, 298 (2013) p. 120.

[3] M. Dao, L. Lu, R. Asaro, J.T.M. De Hosson, E. Ma, Acta Materialia, 55 (2007) p. 4041.

[4] T. Chookajorn, H.A. Murdoch, C.A. Schuh, Science, 337 (2012) p. 951.

[5] Electron microscopy was conducted at the Center for Nanophase Materials Sciences, which is a DOE Office of Science User Facility.

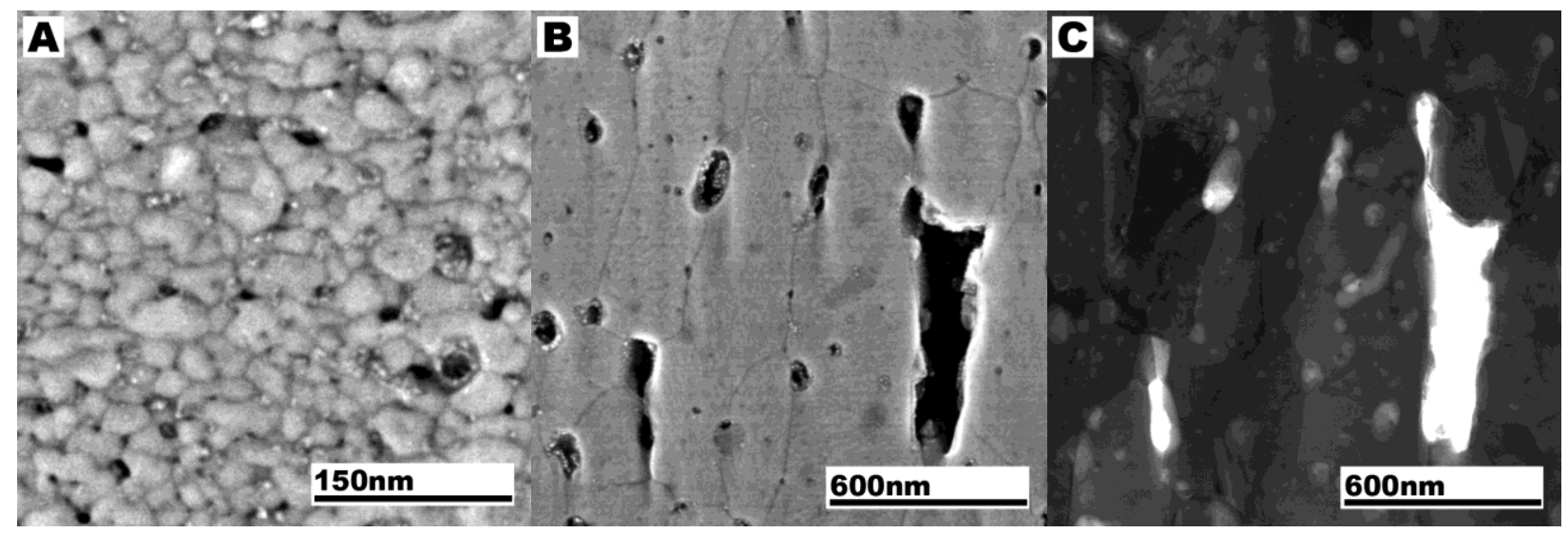

Figure 1. (A) STEM SE micrograph showing equiaxed grains in the as-received nanocrystalline Ag-W . (B) STEM SE micrograph showing the elongated grain structure in the welded material. (C) Grain structure, voids and $\mathrm{WO}_{2}$ dispersoids in the welded material observed in a STEM BF micrograph.
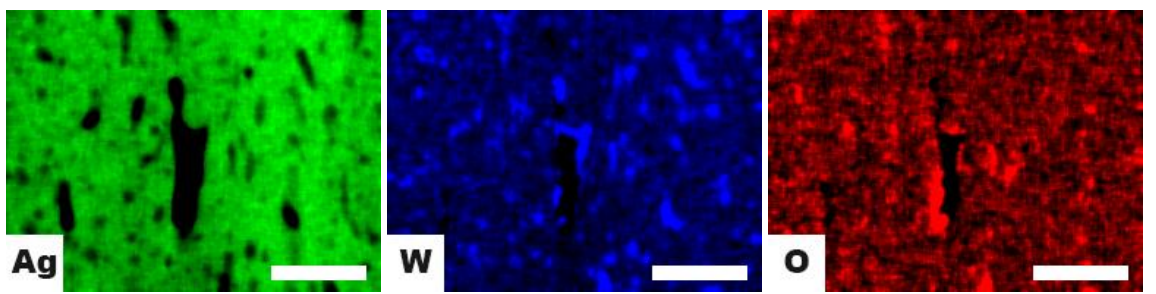

Figure 2. STEM EDS X-ray dot maps show the distribution of $\mathrm{W}$ and $\mathrm{O}$ in the weld region after joining. The contrast in the STEM SE and BF images corresponds with voids and $\mathrm{WO}_{2}$ second phase particles. Scale bar in each image is $700 \mathrm{~nm}$.

Figure 3. (A) FIB lamella used in both the STEM and tKD experiments pictured here. Solid box is the tKD ROI and dashed box is the STEM EDS ROI. (B) Elongated grains in tKD IPF map show no preferential orienation after ultrasonic welding.
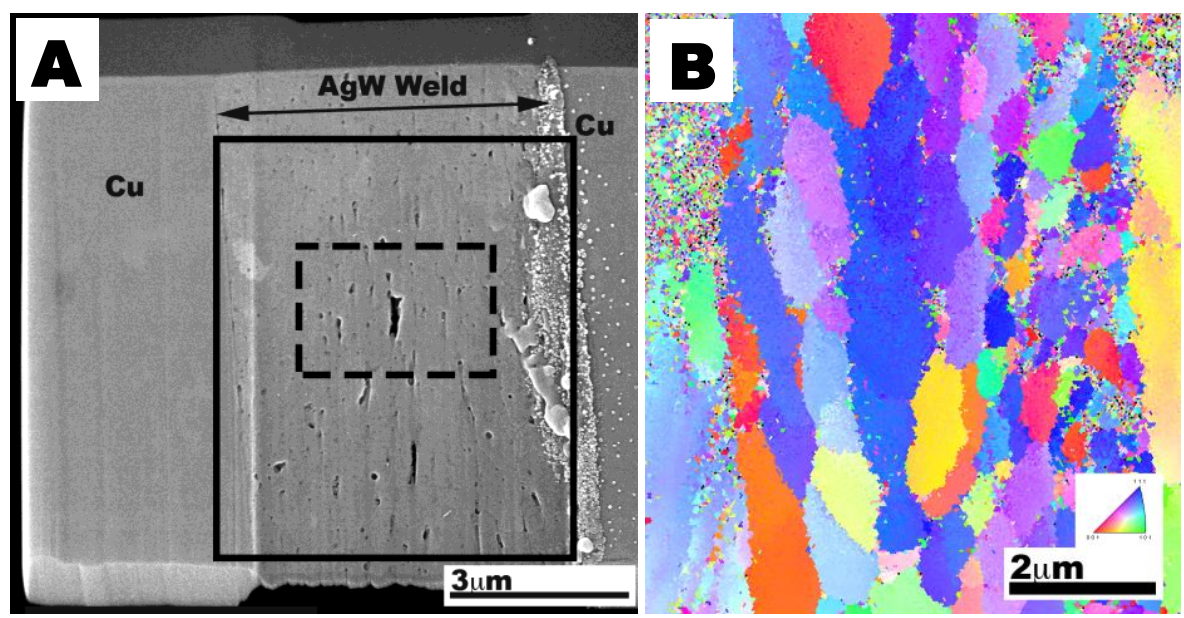